
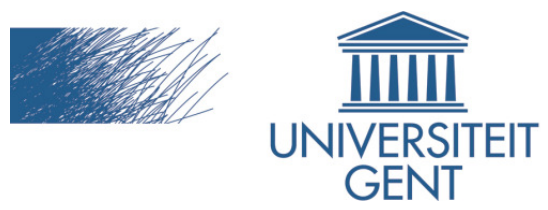

biblio.ugent.be

The UGent Institutional Repository is the electronic archiving and dissemination platform for all UGent research publications. Ghent University has implemented a mandate stipulating that all academic publications of UGent researchers should be deposited and archived in this repository. Except for items where current copyright restrictions apply, these papers are available in Open Access.

This item is the archived peer-reviewed author-version of:

Illumination effects on the differenced Normalized Burn Ratio's optimality for assessing fire severity Veraverbeke, S., Verstraeten, W.W., Lhermitte, S. \& Goossens, R.

In: International Journal of Applied Earth Observation and Geoinformation, Volume 12 (1), 60-70, 2010.

doi: 10.1016/j.jag.2009.10.004

To refer to or to cite this work, please use the citation to the published version:

Veraverbeke, S., Verstraeten, W.W., Lhermitte, S. \& Goossens, R. (2010). Illumination effects on the differenced Normalized Burn Ratio's optimality for assessing fire severity. International Journal of Applied Earth Observation and Geoinformation 12(1), 60-70. doi: 10.1016/j.jag.2009.10.004 


\title{
Illumination effects on the differenced Normalized Burn Ratio's optimality for assessing fire severity
}

\author{
S. VERAVERBEKE* $\dagger$, W.W. VERSTRAETEN§, S. LHERMITTE $\ddagger$ AND R. GOOSSENS $\dagger$ \\ $\dagger$ Department of Geography, Ghent University, Ghent, Belgium \\ $\S$ Geomatics Engineering, Katholieke Universiteit Leuven (K.U.Leuven), Leuven, Belgium \\ † Centro de Estudios Avanzados en Zonas Aridas (CEAZA), Universidad de la Serena, La \\ Serena, Chile \\ *Corresponding author. Email: sander.veraverbeke@ugent.be
}

\begin{abstract}
The influence of illumination effects on the optimality of the dNBR (differenced Normalized Burn Ratio) was evaluated for the case of the 2007 Peloponnese (Greece) wildfires using a pre/post-fire Landsat TM (Thematic Mapper) image couple. Well illuminated pixels (south and south-east facing slopes) exhibited more optimal displacements in the bi-spectral feature space than more shaded pixels (north and north-west exposed slopes). Moreover, pixels experiencing small image-to-image differences in illumination obtained a higher optimality than pixels with a relatively large difference in illumination. To correct for illumination effects, the c-correction method and a modified c-correction technique were applied. The resulting median dNBR optimality of uncorrected, c-corrected and modified c-correction data was respectively $0.58,0.60$ and 0.71 (differences significant for $\mathrm{p}<0.001$ ). The original ccorrection method improved the optimality of badly illuminated pixels while deteriorating the optimality of well illuminated pixels. In contrast, the modified c-correction technique improved the optimality of all the pixels while retaining the prime characteristic of topographic correction techniques, i.e. detrending the illumination-reflectance relationship. For a minority of the data, for shaded pixels and/or pixels with a high image-to-image difference in illumination, the original c-correction outperformed the modified c-correction technique. In this study conducted in rugged terrain and with a bi-temporal image acquisition scheme that substantially deviated from the ideal anniversary date scheme the modified ccorrection technique resulted in a more reliable change detection.
\end{abstract}

Keywords: fire severity, Normalized Burn Ratio, index optimality, Landsat Thematic Mapper, Peloponnese, illumination effect, topographic correction, c-correction

\section{Introduction}

Wildfires play an important role in many ecosystems (Dwyer et al., 1999; Pausas, 2004; Riano et al., 2007) as they partially or completely remove the vegetation layer and affect postfire vegetation composition (Epting and Verbyla, 2005; Lentile et al. 2005; Telesca and Lasaponara, 2006). The fire impact can be described as (i) the amount of damage (Hammill and Bradstock, 2006; Gonzalez-Alonso et al., 2007; Chafer, 2008), (ii) the physical, chemical and biological changes (Landmann, 2003; Chafer et al., 2004; Cocke et al., 2005; Stow et al., 2007; Lee et al., 2008) or (iii) the degree of alteration (Brewer et al., 2005; Eidenshink et al., 2007) that fire causes to an ecosystem and is quantified as the severity of fire. In this context, the terms fire and burn severity are often interchangeably used. Fire severity estimates the short-term fire effect in the immediate post-fire environment (Lentile et al., 2006). An immediate post-fire assessment minimizes the interference with ecosystem's response 
processes (such as vegetation recovery) and it is best suited for emergency rehabilitation plans (Key and Benson, 2005; Eidenshink et al., 2007). Burn severity, on the other hand, quantifies both short- and long-term impact as it includes response processes (Key and Benson, 2005), although the distinction between fire and burn severity has recently become subject for discussion (Keeley, 2009). In this study we focus on fire severity.

Even though a considerable amount of remote sensing studies have focused on the use of the Normalized Difference Vegetation Index (NDVI) for assessing fire severity (Isaev et al., 2002; Diaz-Delgado et al., 2003; Ruiz-Gallardo et al., 2004; Chafer et al., 2004; Hammill and Bradstock, 2006; Hudak et al., 2007), the Normalized Burn Ratio (NBR) has become accepted as the standard spectral index to estimate the severity of fire (e.g. Lopez-Garcia and Caselles, 1991; Epting et al., 2005; Key and Benson., 2005; Bisson et al., 2008). The NBR relates to vegetation structure and moisture by combining near infrared (NIR) and mid infrared (MIR) reflectance and is defined as:

$$
N B R=\frac{T M 4-T M 7}{T M 4+T M 7}
$$

where TM4 and TM7 are respectively the NIR and MIR reflectance of Landsat Thematic Mapper (TM) imagery. Since fire effects on vegetation produce a reflectance increase in the MIR spectral region and a NIR reflectance drop (Pereira et al., 1999), bi-temporal image differencing is frequently applied on pre- and post-fire NBR images resulting in the differenced Normalized Burn Ratio (dNBR) (Key and Benson, 2005). Apart from the correlation with field data (Key and Benson, 2005; De Santis and Chuvieco, 2009), the performance of bi-spectral indices can be evaluated by assessing a pixel's shift in the bispectral feature space. As such, a pixel-based optimality measure, originating from the spectral index theory (Verstraete and Pinty, 1996), has been developed by Roy et al. (2006). They used the optimality concept to question the dNBR method as an optimal fire severity approach. As the optimality approach is pixel-based, it does not suffer from field sampling constraints and, as a consequence, can be applied on all burned pixels. The optimality value varies between zero (not at all optimal) and one (fully optimal). An optimal fire severity spectral index needs to be very sensitive to fire-induced vegetation changes and insensitive to perturbing factors such as atmospheric and illumination effects.

These illumination effects are initiated by both topography and solar position at the moment of image acquisition and influence an object's reflectance behavior. Differences in solar illumination due to topographic position cause a high variation in reflectance response for similar terrain features: well illuminated areas show higher reflectance values than expected, whereas in shaded areas reflectance is typically lower (Leprieur et al., 1988). Topographic effects consequently become more obvious in more rugged terrain. They influence any image processing technique based on individual band reflectance, such as Land Use Land Cover (LULC) classifications (Bishop and Colby, 2002; Riano et al. 2003; Mitri and Gitas, 2004). Hence, a range of topographic normalization techniques have been developed with the prime goal to detrend the illumination-reflectance relationship (Teillet et al., 1982; Civco, 1989; Vincini and Frazzi, 2003; Kobayashi and Sanga-Ngoie, 2008). Topographic effects in ratioimages based analysis, however, are assumed to be minimal (Song and Woodcock, 2003) and are not considered in most studies using the NBR to assess fire severity. Key (2005) stated that poor illumination and increased shadow decreases the definition of fire effects and sharpness of dNBR images. Verbyla et al. (2008) showed that topography clearly affects both NBR and dNBR values. They simulated the incoming solar radiation and found a decreasing trend in post-fire NBR while insolation increased, whereas NIR reflectance, MIR reflectance 
and dNBR values increased with increasing insolation. Therefore, the focus of this research is to quantitatively evaluate illumination effects due to topography using the pixel-based optimality measure and to propose a topographic correction approach to become a more reliable fire severity assessment using the dNBR. This general objective is fulfilled by (i) disclosing the effect of illumination conditions on index performance in topographically uncorrected images, (ii) evaluating the effect of topographic correction on individual band reflectance, and (iii) evaluating the effect of topographic correction on the dNBR optimality.

\section{Methods}

\subsection{Study area}

The study area is situated at the Peloponnese peninsula, in southern Greece $\left(36^{\circ} 30^{\prime}-38^{\circ} 30^{\prime} \mathrm{N}\right.$, $21^{\circ}-23^{\circ} \mathrm{E}$ ) (see figure 1). The topography is rugged with elevations ranging between 0 and $2404 \mathrm{~m}$ above sea level. The climate is typically Mediterranean with hot, dry summers and mild, wet winters (see figure 2). For the Kalamata meteorological station ( $37^{\circ} 4^{\prime} \mathrm{N}, 22^{\circ} 1^{\prime} \mathrm{E}$ ) the average annual temperature is $17.8^{\circ} \mathrm{C}$ and the mean annual precipitation equals $780 \mathrm{~mm}$.

After a severe drought period several large wildfires of unknown cause have struck the area in the 2007 summer. The first large burn initiated at 26/07/2007 and the burns prolonged till 01/09/2007. The fires consumed a large amount (more than 115000 ha) of coniferous forest, broadleaved forest, shrub lands (maquis and phrygana communities) and olive groves. Black pine (Pinus nigra) is the dominant conifer species. Maquis communities consist of sclerophyllous evergreen shrubs of 2-3 m high (Polunin 1980). Phrygana is dwarf scrub vegetation $(<1 \mathrm{~m})$, which prevails on dry landforms (Polunin 1980). The shrub layer is characterised by e.g. Quercus coccifera, Q. frainetto, Pistacia lentiscus, Cistus salvifolius, C. incanus, Erica arborea, Sarcopoterum spinosum. The olive groves consist of Olea europaea trees, whereas oaks are the dominant broadleaved species.

\subsection{Data and preprocessing}

For assessing fire severity of the summer 2007 Peloponnese fires the best available pre/postfire Landsat TM image couple (path/row 184/34) was chosen. The first cloud-free post-fire image was acquired at 28/09/2007 (solar elevation $46.43^{\circ}$, solar azimuth $150.18^{\circ}$ ). The prefire image was chosen based on anniversary date acquisition, data quality and cloudiness. The pre-fire image dates from 23/07/2006 (solar elevation $62.89^{\circ}$, solar azimuth $123.30^{\circ}$ ). Both images are acquired in the Mediterranean summer drought period, which prolongs from June till September (see figure 2), and as such phenological differences are assumed to be minimal. The images were subjected to geometric, radiometric and atmospheric correction.

The 2007 image was geometrically corrected using 34 ground control points (GCPs), recorded in the field with a Garmin eTrex Vista GPS (Global Positioning System) (5-10 m error in $x$ and y). The resulting Root Mean Square Error (RMSE) was lower than 0.5 pixels. The 2006 and 2007 images were co-registered within 0.5 pixels accuracy. All images were registered in Universal Transverse Mercator (zone 34S), with ED 50 (European Datum 1950) as geodetic datum. Topographical slope and aspect data were derived from $90 \mathrm{~m}$ SRTM (Shuttle Radar Topography Mission) elevation data (Jarvis et al., 2006) resampled to and co-registered with the Landsat images.

Raw digital numbers (DNs) of the were scaled to at-sensor radiance values $\left(\mathrm{L}_{\mathrm{s}}\right)(\mathrm{Chander}$ et al. 2007) but with band-specific parameters proposed for Landsat TM data processed and 
distributed by the ESA (European Space Agency) (Arino et al. s.d.). The radiance to reflectance conversion was performed using the COST method of Chavez (1996):

$\rho_{a}=\frac{\pi\left(L_{s}-L_{d}\right)}{\left(E_{o} / d^{2}\right)\left(\cos \theta_{z}\right)^{2}}$

where $\rho_{a}$ is the atmospherically corrected reflectance at the surface; $L_{s}$ is the at-sensor radiance $\left(\mathrm{Wm}^{-2} \mathrm{sr}^{-1}\right) ; L_{d}$ is the path radiance $\left(\mathrm{Wm}^{-2} \mathrm{sr}^{-1}\right) ; E_{o}$ is the solar spectral irradiance $\left(\mathrm{Wm}^{-2}\right) ; d$ is the earth-sun distance (astronomical units); and $\theta_{z}$ is the solar zenith angle. The COST method is a dark object subtraction (DOS) approach that assumes $1 \%$ surface reflectance for dark objects (e.g. deep water). After applying the COST atmospheric correction, pseudo-invariant features (PIFs), such as deep water and bare soil pixels, were evaluated in the images and no further relative normalization between the images was considered.

As this study focuses exclusively on burned pixels, a burned area mask was created. This was done using a two-phase dNBR threshold (Garcia and Chuvieco, 2004). To avoid possible confusion with harvested crop land a rough fire perimeter (approximately $1 \mathrm{~km}$ outside the burned area) was manually digitized. Then, during the first phase, severely burned pixels were selected using a threshold of $\mathrm{dNBR}>0.4$. During the second phase a 15 by 15 pixels contextual algorithm was initiated with the core burn pixels as centers using a more relaxed dNBR $>0.1$ threshold. The first phase reduces commission errors, while the second phase reduces omission errors (Chuvieco et al., 2008). The accuracy of the burned area mask is evaluated with the probability of detection and the probability of false alarm statistics (Giglio et al., 2008) using 242 reference sample points visited in the field and GPS-recorded in September 2008 (143 burned samples and 99 unburned samples in close vicinity of the burned area). The burned area map has a detection probability of $80 \%$ and a probability of false alarm of $5 \%$. In total 1282779 pixels were discriminated equaling approximately 115000 ha.

\section{3 dNBR optimality}

For evaluating the optimality of the bi-temporal change detection the TM4-TM7 bi-spectral space was considered (see figure 3). If a spectral index is appropriate to the physical change of interest, in this case fire-induced vegetation depletion, there exist a clear relationship between the change and the direction of the displacement in the bi-spectral feature space (Verstraete and Pinty, 1996). In an ideal scenario a pixel's bi-temporal trajectory is perpendicular to the first bisector of the Cartesian coordinate system. This is illustrated in figure 3 for the displacement from unburned $(\mathrm{U})$ to optimally $(\mathrm{O})$ sensed burned. Perturbing factors such as atmosphere and illumination decrease the performance of the index. Then a pixel's displacement can be decomposed in a vector perpendicular to the first bisector and a vector along the post-fire NBR isoline to which the index is insensitive. For example, in figure 3, a pixel shifts from unburned (U) to burned (B) after fire. Here, the magnitude of change to which the index is insensitive is equal to the Euclidian distance $|O B|$. Thus the observed displacement vector UB can be decomposed in the sum of the vectors UO and OB, hence, following the expression of Roy et al. (2006) the index optimality is defined as:

optimality $=1-\frac{|O B|}{|U B|}$ 
As $|O B|$ can never be larger than $|U B|$, the optimality measure varies between zero and one. If the optimality measure equals zero, then the index is completely insensitive to the change of interest. An optimality score of one means that the index performs ideally.

\subsection{Correcting for illumination effects}

A variety of topographic correction techniques have been proposed in literature. Most of these methods correct for illumination effects based on the cosine of the incidence angle $\gamma_{i}$, which is the angle between the normal to the ground and the sun rays, using a digital elevation model (DEM) and knowledge of the solar zenith and azimuth angle at the moment of image acquisition (Teillet et al., 1982):

$$
\cos \gamma_{i}=\cos \theta_{p} \cos \theta_{z}+\sin \theta_{p} \sin \theta_{z} \cos \left(\phi_{a}-\phi_{o}\right)
$$

where $\gamma_{i}$ is the incident angle; $\theta_{p}$ is the slope angle; $\theta_{z}$ is the solar zenith angle; $\phi_{a}$ is the solar azimuth angle; and $\phi_{o}$ is the aspect angle. The illumination parameter $\cos \gamma_{i}$ ranges between minus one to one.

Teillet et al. (1982) introduced the cosine correction method by multiplying original reflectance values by the factor $\cos \theta_{z} / \cos \gamma_{i}$. Unfortunately, this Lambertian method overcorrects the image, especially in low illumination areas (Meyer et al., 1993; Riano et al., 2003). To account for the overcorrection in the cosine method non-Lambertian semi-empirical techniques have been proposed, i.e. the Minnaert correction (Colby and Keating, 1998; Bishop and Colby, 2002) and the c-correction (Teillet et al., 1982). In this study we focus on the c-correction as this method has proven to give satisfying results in a whole range of applications (Meyer et al., 1993; Riano et al., 2003, Kobayashi and Sanga-Ngoie, 2008, Vicente-Serrano et al., 2008).

In the c-correction method terrain corrected reflectance $\rho_{t}$ is defined as:

$\rho_{t}=\rho_{a}\left(\frac{\cos \theta_{z}+c_{k}}{\cos \gamma_{i}+c_{k}}\right)$

Where $c_{\mathrm{k}}$ is a band specific parameter $c_{k}=b_{k} / m_{k}$ where $b_{\mathrm{k}}$ and $\mathrm{m}_{\mathrm{k}}$ are the respective intercept and slope of the regression equation $\rho_{a}=b_{k}+m_{k} \cos \gamma_{i}$.

In the original c-correction technique (see equation 5) terrain corrected reflectance $\rho_{t}$ is lower than the original reflectance $\rho_{a}$ when $\cos \gamma_{i}$ is larger than $\cos \theta_{z}$ as then the correction factor $\cos \theta_{z}+c_{k} / \cos \gamma_{i}+c_{k}$ becomes smaller than one. If $\cos \gamma_{i}$ is smaller than $\cos \theta_{z}$, terrain corrected reflectance $\rho_{t}$ is higher than the original reflectance $\rho_{a}$ as the correction factor then becomes larger than one. Thus the original c-correction technique normalizes reflectance depending on the solar zenith. However, remote sensing conditions are likely to be optimal at maximum illumination. Therefore we propose a modified c-correction method that corrects reflectance to a maximum illumination $\cos \gamma_{i}$ of one, instead of normalizing as a function of the solar zenith angle: 


$$
\rho_{t}=\rho_{a}\left(\frac{1+c_{k}}{\cos \gamma_{i}+c_{k}}\right) .
$$

\subsection{Analysis}

The effect of the topographic correction is evaluated both in terms of individual band reflectance and in terms of dNBR optimality. The prime goal of any topographic correction technique is to detrend the illumination-reflectance relationship (Meyer et al., 1993; Ekstrand, 1996, Soenen et al., 2008). Original reflectance values $\rho_{a}$ increase with increasing illumination $\cos \gamma_{i}$. Topographic correction techniques should reduce the slope of the regression between these two variables (Meyer et al., 1993).

Additionally, the effect of the topographic correction techniques is evaluated by comparing median index optimality of topographically corrected and uncorrected data among different aspect, slope, average illumination $\cos \gamma_{i, \text { avg }}$ and difference in illumination $\cos \gamma_{i, \text { diff }}$ classes. The median is used because the optimality distributions functions appeared to be non-normal and because of its robustness in the presence of outlier values.

In rugged terrain the degree of incoming solar radiation is largely determined by the topographical parameters aspect angle $\phi_{o}$ and slope angle $\theta_{p}$. Therefore, uncorrected and corrected dNBR optimality were compared among ten equal interval slope classes and eight different aspect classes: $\mathrm{N}\left(\phi_{o}>337.5^{\circ}\right.$ or $\left.\phi_{o}<22.5^{\circ}\right)$, NE $\left(22.5^{\circ}<\phi_{o}<67.5^{\circ}\right)$, E $\left(67.5^{\circ}<\right.$ $\left.\phi_{o}<112.5^{\circ}\right)$, SE $\left(112.5^{\circ}<\phi_{o}<157.5^{\circ}\right), \mathrm{S}\left(157.5^{\circ}<\phi_{o}<202.5^{\circ}\right), \mathrm{SW}\left(202.5^{\circ}<\phi_{o}<\right.$ $\left.247.5^{\circ}\right), \mathrm{W}\left(247.5^{\circ}<\phi_{o}<292.5^{\circ}\right), \mathrm{NW}\left(292.5^{\circ}<\phi_{o}<337.5^{\circ}\right)$.

When the acquisition parameters (the solar zenith angle $\theta_{z}$ and the solar azimuth angle $\phi_{a}$ ) are also considered, the average illumination $\cos \gamma_{i, \text { avg }}$ of the 2006 and 2007 images can be defined as:

$\cos \gamma_{i, a v g}=\frac{\cos \gamma_{i, 2006}+\cos \gamma_{i, 2007}}{2}$

The difference in illumination $\cos \gamma_{i, d i f f}$ between the pre-and post-fire image is calculated as:

$\cos \gamma_{i, d i f f}=a b s\left(\cos \gamma_{i, 2006}-\cos \gamma_{i, 2007}\right)$.

We define average illumination $\cos \gamma_{i, \text { avg }}$ and difference in illumination $\cos \gamma_{i, \text { diff }}$ as we want to account for both overall illumination conditions $\left(\cos \gamma_{i, a v g}\right)$ and the difference in illumination between the two images $\left(\cos \gamma_{i, d i f f}\right)$. Pixels were first aggregated in ten equal interval classes of average illumination $\cos \gamma_{i, a v g}$ and difference in illumination $\cos \gamma_{i, d i f f}$. In the second step index optimality of topographically uncorrected and corrected data was compared among the different classes.

3 Results

\subsection{Influence on individual band reflectance}


Detrending the illumination-reflectance relationship ideally would lead to a coefficient of determination $\left(\mathrm{R}^{2}\right)$ of zero. This means that the zero hypothesis that the slope of the regression line is equal to zero could no longer be rejected. Table 1 summarizes slope, intercept and $\mathrm{R}^{2}$ of the various regression models with illumination as independent variable and uncorrected or corrected reflectance as dependent variable. The $\mathrm{R}^{2}$ values of the uncorrected bands show a moderate-low correlation between illumination and reflectance. This correlation was clearly higher for the uncorrected post-fire bands $\left(\mathrm{R}^{2}=0.14\right.$ for TM4 2007 and $\mathrm{R}^{2}=0.29$ for TM7 2007) in comparison with the uncorrected pre-fire bands $\left(\mathrm{R}^{2}=\right.$ 0.02 for TM4 2006 and TM7 2006). After applying both topographic correction methods (equations 5 and 6) the $\mathrm{R}^{2}$ statistic approximated zero in all cases $\left(\mathrm{R}^{2}<0.001\right)$. The slope of the regression models also decreased for the four studied bands for both topographic correction methods. Again this decrease is more explicit for the post-fire bands. The slope of the regression models approximated zero after correction. The intercept parameter is higher after applying a topographic correction technique. This feature is slightly more apparent for the modified c-correction than for the original c-correction technique, especially for the 2007 image.

\subsection{Influence on dNBR optimality}

\subsubsection{Overall performance}

Figures 4A-C depict the topographically uncorrected, c-corrected and modified c-correction dNBR optimality maps of the burned areas. The modified c-correction dNBR optimality (median $=0.71)$ outperformed c-corrected and uncorrected optimality (medians of respectively 0.60 and 0.58 ), whereas c-corrected optimality provided slightly better results than uncorrected optimality. This is also reflected when the respective histograms are inspected (see figures 4D-F). According to the non-parametric Wilcoxon test (Hollander and Wolfe, 1999) differences in median optimality and dNBR optimality distribution functions are statistically significant $(\mathrm{p}<0.001)$. Most of the pixels have uncorrected dNBR optimality values higher than 0.3 and the histogram peaks for values between 0.3 and 0.4 . Also for the ccorrected data most of the pixels have optimality scores higher than 0.3 , although these pixels are evenly distributed between values of 0.3 and 1.0. In contrast, in the modified c-correction optimality histogram the number of pixels steadily increased with increasing optimality.

\subsubsection{Influence of topographical parameters}

Among different aspect classes (see figure 5A), south-east and south facing slopes yielded the highest optimality score (0.70), when comparing median dNBR optimality of uncorrected data. South-west and east oriented slopes had slightly lower median optimality values of respectively 0.65 and 0.64 . Median optimality scores decreased even more for north-east and west facing slopes (respectively 0.54 and 0.56 ). The worst index performance was obtained for north and north-west sloping areas with median optimality measures of respectively 0.40 and 0.42 . The original c-correction technique improved the optimality of west, north-west, north and north-east oriented pixels, while deteriorating the optimality of east, south-east, south and south-west oriented pixels. In contrast the modified c-correction technique ameliorated the optimality of all aspect orientations yielding optimality scores between 0.68 and 0.74 for all aspect orientations. For the modified c-correction, more southerly exposed pixels kept a slightly higher optimality in comparison with more northerly exposed slopes, however, differences became smaller than these observed for the uncorrected data.

Among the different topographical slope classes (see figure 5B) the optimality statistic of uncorrected data remained more or less constant between 0.56 and 0.60 between 0 and $40^{\circ}$. 
The optimality of the uncorrected data suddenly dropped for the steepest slopes $\left(>40^{\circ}\right)$, e.g. the optimality of the $>45^{\circ}$ slope class equaled 0.36 . The original c-correction technique resulted in approximately the same optimality values as the uncorrected data for slopes between 0 and $25^{\circ}$. For steeper slopes $\left(>25^{\circ}\right)$, the original c-correction clearly improved the optimality. The steeper the slopes, the more explicit this phenomenon was. In comparison with the uncorrected and c-corrected data, the modified c-correction technique ameliorated the optimality of all the slope classes, except for the slope classes $>40^{\circ}$. For these two classes, representing $2.20 \%$ of the total number of pixels, the original c-correction technique performed better. For all the other classes, optimality raised to values between 0.70 and 0.73 after applying the modified c-correction technique.

\subsubsection{Influence of illumination geometry}

When looking at the average illumination $\cos \gamma_{i, a v g}$ (see figure 6A) a consistent increasing trend in median dNBR optimality of uncorrected data was observed as the average illumination $\cos \gamma_{i, a v g}$ class varied from 0.0 to 1.0. There were only few pixels experiencing very low average illumination $(0.01 \%$ of the total number of pixels in the $0.0-0.1$ class, $0.10 \%$ in the $0.1-0.2$ class and $0.35 \%$ in the $0.2-0.3$ class). These classes yielded very low median optimality scores (e.g. median dNBR optimality for the $0.0-0.1$ class equals 0.30 ). In contrast, most of the pixels were quite well illuminated $(14.41 \%$ of the total number of pixels in the $0.6-0.7$ class, $33.39 \%$ in the $0.7-0.8$ class, $34.32 \%$ in the $0.8-0.9$ class and $8.13 \%$ in the $0.9-$ 1.0 class) and these pixels' optimality increased substantially peaking in a median dNBR optimality score of 0.73 for the best illuminated class. Optimality of the average illumination $\cos \gamma_{i, a v g}$ classes between 0.0 and 0.8 rose for both c-corrected and modified c-correction data (up to 0.32 for $\cos \gamma_{i, a v g}$ between 0.2 and 0.4). However, for the well illuminated $\cos \gamma_{i, a v g}$ classes between 0.8 and 1.0 optimality diminished for c-corrected data, while the modified ccorrection method produced a slightly better optimality in comparison with the uncorrected data (see figure 6A). The majority of the pixels (approximately $96 \%$ of the total number of pixels) experienced a relatively high average illumination $\cos \gamma_{i, a v g}$ between 0.5 and 1.0. For these pixels the modified c-correction technique yielded higher optimality values than the original c-correction method. In contrast with this, the original c-correction technique outperformed the modified c-correction technique for a minority of pixels, representing approximately $4 \%$ of the data, which experienced a relatively low average illumination $\cos \gamma_{i, a v g}$ between 0.0 and 0.5 .

For the difference in illumination $\cos \gamma_{i, \text { diff }}$ (see figure 6B) median dNBR optimality of uncorrected data also showed a consistent decreasing trend as the difference in illumination $\cos \gamma_{i, \text { diff }}$ varied from 0.0 to 1.0. The majority of the pixels experienced only slightly differing illumination conditions ( $30.53 \%$ of the total number of pixels in the $0-0.1$ class, $33.39 \%$ in the 0.1-0.2 class, $19.25 \%$ in the $0.2-0.3$ class and $9.03 \%$ pixels in the $0.3-0.4$ class). These pixels produced high median optimality scores, e.g. the class with the lowest difference in illumination yielded the highest median optimality value (0.69). As difference in illumination increased, the number of pixels per class and the median optimality value decreased leading to a low median optimality measure of 0.31 for the $0.03 \%$ of the total number of pixels in the 0.9-1.0 class. A similar trend as observed in figure $6 \mathrm{~A}$ can be deduced from figure $6 \mathrm{~B}$. There, both correction methods improve the optimality of the difference in illumination $\cos \gamma_{i, \text { diff }}$ classes between 0.2 and 1.0 compared to uncorrected data (up to an increase of 0.36 for 
$\cos \gamma_{i, \text { diff }}$ between 0.7 and 0.8). For those pixels that experienced little illumination differences ( $\cos \gamma_{i, \text { diff }}$ classes between 0.0 and 0.2) c-corrected data produced a decrease in optimality, while the modified c-correction technique resulted in pixels with higher optimality values compared to the uncorrected data. Again, for approximately $96 \%$ of the data represented in the $\cos \gamma_{i, \text { diff }}$ classes between 0.0 and 0.5 , the modified c-correction technique excelled the original c-correction technique. In contrast, for a minority of pixels with a relatively high difference in illumination $\cos \gamma_{i \text {,diff }}$ (approximately $4 \%$ of the data, representing the $\cos \gamma_{i, \text { diff }}$ classes between 0.5 and 1.0) the original c-correction gave better results than the modified c-correction.

\section{Discussion}

\subsection{Influence on individual band reflectance}

Reflectance is known to increase with increasing cosine of the incidence angle for similar terrain features (Soenen et al., 2008; Wu et al., 2008). Ekstrand (1996) showed that the TM4 and TM5 bands were most affected by topographic effects expressed in a non-linear, and thus non-Lambertian, response to increasing illumination. For TM2, 3 and 7 this non-linearity was not that evident, although these bands exhibited a statistically significant relationship with topography. TM1 was the only band in the study of Ekstrand (2006) where topography had no clear effect. The c-correction technique and other topographic correction techniques have proven to effectively remove the trend of increasing reflectance with increasing illumination (Meyer et al., 1993; Vincini and Frazzi, 2003; Huang et al., 2008; Soenen et al., 2008; Wu et al., 2008). This characteristic feature of topographic correction technique remained unaffected in our modified version of the c-correction method.

The correlation between uncorrected reflectance and illumination was higher for the post-fire bands than for the pre-fire bands. This can be explained by the fact that after the fire a more homogeneous land cover, i.e. burned land, is introduced in the analysis. The pre-fire bands consist of a heterogeneous mixture of land cover types. This might be the reason why the expected relationship between uncorrected reflectance and illumination is rather weak by the pre-fire bands. A more heterogeneous land cover generally results in lower $\mathrm{R}^{2}$ for the uncorrected reflectance-illumination regression (Bishop and Colby, 2002). The same holds true when the post-fire slope parameter of the regression equation is considered. The slope parameter is higher for the uncorrected post-fire bands than for the uncorrected pre-fire bands, revealing a stronger relation between reflectance and illumination for the uncorrected post-fire bands. As topographic correction techniques are known to work better when applied on homogeneous land covers (Bishop and Colby, 2002) the decrease in the $\mathrm{R}^{2}$ statistic and slope parameter is more obvious for the post-fire bands. Another important feature observed is that both topographic correction techniques increase the intercept parameter. This is trivial since the direct proportional relation between reflectance and illumination as observed by the uncorrected bands is ruled out towards an approximately horizontal regression line after applying a topographic correction. As a consequence the intersection with the y-axis (reflectance) is at a higher reflectance value. However, a difference between the two correction techniques is observed in the intercept parameter. The modified c-correction technique results in a slightly higher intercept value than the original c-correction technique. This might be expected as the modified method corrects to a maximum illumination condition instead of normalizing to an intermediate illumination condition as the original method does. 
This difference again is more obvious for the post-fire bands than for the pre-fire bands, probably as a consequence of the more homogeneous post-fire land cover.

\subsection{Influence on dNBR optimality}

Previous studies have analyzed the dNBR's optimality for assessing fire severity without performing topographic corrections, i.e. uncorrected dNBR data (Roy et al., 2006; Escuin et al., 2008; Murphy et al., 2008). In this study, the median uncorrected dNBR optimality equaled 0.58 . This approximates to average optimality values reported by Escuin et al. (2008) (0.49) and Murphy et al. (2008) (range: 0.26-0.80 for six burns in Alaska, USA). Results, however, contrast with the very low mean dNBR optimality scores $(0.1)$ reported by Roy et al. (2006) based on Landsat ETM+ imagery for African savannah burns. These authors also report low dNBR optimality values for MODIS sensed fires in other ecosystems (Russia, Australia and South America). These results suggest that the dNBR index is suboptimal for assessing fire severity. The poor optimality results obtained by Roy et al. (2006) can possibly be explained by the fact that the authors also included unburned pixels in their analysis. Unaffected pixels are generally associated with low optimality scores since a pixel's shift in the bi-spectral space is then only caused by noise (Escuin et al., 2008).

It is generally assumed that in ratio-based images the role of topography is ruled out (e.g. Song and Woodcock, 2003). This is true to some degree, however, when analysis is conducted in rugged terrain with low sun angle imagery, illumination effects cannot longer be neglected. Key (2005) qualitatively indicated the importance of illumination conditions for discriminating burned areas in dNBR maps as low reflectance effectively degraded burned and unburned qualities. Verbyla et al. (2008) evaluated the illumination effect on index values. They found pre-and post-fire NBR values differing among south and north facing slopes. The influence of slope exposition, however, was ambiguous as the direction of the differences was opposite for two burns. These authors also found a clear NIR, MIR and dNBR values increase with increasing insolation. In opposite, the post-fire NBR consistently decreased with increasing insolation. This research quantitatively pointed out the importance of illumination effects in a dNBR-based fire severity assessment and indicated that this may influence the reliability of the dNBR approach.

The pixel-based optimality measure has given a unique opportunity to assess the influence of illumination on the reliability of a topographically uncorrected dNBR-based fire severity assessment. With respect to the solar azimuth angles of the two images in our study $\left(\phi_{a}=\right.$ $123.3^{\circ}$ for the 2006 image and $\phi_{a}=150.18^{\circ}$ for the 2007 image), we expect south and southeast facing slopes to have the highest optimality. This was observed (see figure 5A) with a resulting median optimality of 0.70 . Consequently, we expect north and north-west exposed slopes to have the smallest optimality, i.e. respectively 0.40 and 0.42 . North-east, east, southwest and west facing areas scored intermediate. With regards to slope steepness the optimality reached a more or less constant value when analyzing dNBR optimality among different topographic slope classes. For the steepest slope classes, however, a clear optimality decrease was observed (see figure 5B). This seems logical as the steeper a pixel's slope is, the higher the probability that the pixel is badly illuminated, especially when the pixel is not exposed to the incoming light. However, following this rationale we would expect a decreasing trend in optimality when slopes become steeper. This trend is apparently not visible in the results. This might be explained by the fact that for slopes lower than $40^{\circ}$ the lower optimality of the badly illuminated pixels is averaged out by the higher optimality of those pixels that are well illuminated. An analogous conclusion as from the aspect analysis can be drawn when median optimality is evaluated among average illumination classes (see figure 6A). Well illuminated 
pixels had a more reliable dNBR value than more shaded pixels. Thus, the introduced noise in the pre/post-fire pixel displacement (i.e. the distance $|O B|$ in figure 3) becomes more important when illumination deteriorates. Additionally, since illumination geometry can differ significantly in the images, the reliability of the change detection can be influenced by these different illumination conditions. In our case study both solar azimuth $\left(\phi_{a}=123.3^{\circ}\right.$ for the 2006 image and $\phi_{a}=150.18^{\circ}$ for the 2007 image $)$ and solar elevation $\left(62.89^{\circ}\right.$ for the 2006 image and $46.43^{\circ}$ for the 2007 image) differed reasonably. This resulted in very low optimality scores for pixels with highly differing illumination (see figure 6B). It should be noted, however, that only few pixels experienced large differences in illumination. In opposite, for the majority of the pixels differences in illumination were minor and these pixels were associated with high median optimality scores.

As remote sensing conditions are assumed to be optimal at maximum illumination, we proposed a modified version of the c-correction technique that correct towards a maximum illumination condition. Both topographic correction techniques, the original and the modified c-correction, possess the prime characteristic of topographic correction methods, i.e. detrending the illumination-reflectance relationship (see 3.1). The dNBR optimality of topographically corrected data outperformed the uncorrected optimality (median $=0.58$ ), although the median optimality of the modified c-correction $(0.71)$ was clearly higher than the median optimality of the original c-correction (0.60). The reason why the difference between the uncorrected and c-corrected data is so little can be deduced from the figures 6A-B. In figure $6 \mathrm{~A}$ it is observed that for the average illumination $\cos \gamma_{i, \text { avg }}$ classes between 0.0 and 0.8 , which represent approximately $58 \%$ of the data, the original c-correction technique realized better optimality scores than the uncorrected data. However, for the approximately $42 \%$ of pixels belonging to the average illumination $\cos \gamma_{i, \text { avg }}$ classes between 0.8 and 1.0, the original c-correction technique underperformed in comparison with uncorrected data. The reason why the original c-correction technique achieved unsatisfying results in the high average illumination $\cos \gamma_{i, \text { avg }}$ classes probably is that those pixels experienced a reflectance decline in comparison with the uncorrected data as the correction factor $\cos \theta_{z}+c_{k} / \cos \gamma_{i}+c_{k}$ in equation 5 became smaller than one. Apparently, this resulted in a lower optimality score for those pixels. Figure 6B depicts an analogous but even more striking characteristic: the original c-correction techniques produced higher optimality scores than uncorrected data for the $36 \%$ of the total number of pixels belonging to the difference in illumination $\cos \gamma_{i, \text { diff }}$ classes varying from 0.2 till 1.0 whereas the optimality of the uncorrected data outperformed the c-corrected optimality for the remaining $64 \%$ of the pixels. In the overall performance (see figure 4) the benefits of the original c-correction technique outweighed its drawbacks resulting in a slightly higher median optimality than for uncorrected data.

In contrast with the undesirable feature of the original c-correction, the modified c-correction improved the optimality of all the pixels in respect to the uncorrected data. The median optimality of the modified c-correction $(0.71)$ is markedly higher than median optimality of the data after original c-correction (0.60). However, an important discrepancy can be inferred from figures $6 \mathrm{~A}-\mathrm{B}$. Figure $6 \mathrm{~A}$ shows that for the high average illumination classes $\cos \gamma_{i, a v g}$ between 0.5 and 1.0, which represent $96 \%$ of the data, the modified c-correction technique outperformed the original c-correction technique. For the low average illumination $\cos \gamma_{i, a v g}$ classes between 0.0 and 0.5 , however, the original c-correction techniques realized better 
optimality scores. Nevertheless, these classes represent only $4 \%$ of the data. Figure 6B depicts an analogous feature. Here, for the low difference in illumination $\cos \gamma_{i, \text { diff }}$ classes between 0.0 and 0.5 , representing $96 \%$ of the data, the modified c-correction produced better optimality scores than the original c-correction. Again a minority of $4 \%$ of the pixels, belonging to the difference in illumination $\cos \gamma_{i, \text { diff }}$ classes between 0.5 and 1.0, experienced a better optimality score with the original c-correction than with the modified c-correction. Overall the advantages of the modified c-corrections dominate its disadvantages. As a consequence the modified c-correction technique realized a clearly higher median optimality than the original c-correction.

However, the deviant behavior of a minority of pixels poses questions on the utility of the modified c-correction for image couples with other illumination differences than our example image couple. In this context, an important recommendation when doing bi-temporal change detection is that the image couple should approximate as closely as possible the anniversary date acquisition scheme (Coppin et al., 2004). This diminishes illumination differences and phenological dissimilarities. But, because of the importance of acquiring the first available cloud-free post-fire image to assess fire severity, bi-temporal acquisition schemes potentially diverge from the ideal anniversary data scheme. After acquiring the first available cloud-free post-fire image, the pre-fire image is often chosen based on data quality and acquisition date. The anniversary date condition is often only partly fulfilled, as in our case study. Therefore, the effect of differing illumination is likely to be more explicit in fire severity applications than in other change detection studies (Key, 2005). Our images diverge more than two months from the anniversary date acquisition scheme (pre-fire image of 23/07/2006 and post-fire image of 28/09/2007) resulting in reasonably high differences in solar azimuth $\left(\phi_{a}=123.3^{\circ}\right.$ for the 2006 image and $\phi_{a}=150.18^{\circ}$ for the 2007 image) and solar elevation $\left(62.89^{\circ}\right.$ for the 2006 image and $46.43^{\circ}$ for the 2007 image). Figure 6B allows reasoning about the performance of the topographic correction techniques when illumination geometry would be different than in our case study. The illumination geometry of two images will be more similar than in our case study when (i) the study area is situated at lower latitude, (ii) topography is less rugged and (iii) the anniversary date scheme is more closely approximated. In this case the number of pixels in the high average illumination $\cos \gamma_{i, a v g}$ and low difference in illumination $\cos \gamma_{i, \text { diff }}$ becomes more important. These classes are characterized by a high optimality score after applying the modified c-correction, in contrast with the clearly lower optimality scores when the original c-correction is applied. In addition, the number of pixels in the low average illumination $\cos \gamma_{i, \text { avg }}$ and high difference in illumination $\cos \gamma_{i, \text { diff }}$ classes reduces. These classes are typified by the better performance of the original c-correction in comparison with the modified c-correction. Thus, when the illumination geometry of the two images is more similar the benefits of the modified c-correction become even more substantial. In opposite, the illumination geometry of two images will be more dissimilar than in our study when (i) the area of interest is located at higher latitude, (ii) topography is more rugged and (iii) there is a more extreme divergence from the anniversary date acquisition scheme. This has a consequence that the number of pixels for which the original c-correction technique performs better becomes larger (for both the low average illumination $\cos \gamma_{i, a v g}$ and high difference in illumination $\cos \gamma_{i, \text { diff }}$ classes). In our example study the original ccorrection method performed better for only $4 \%$ of the data. When illumination conditions differ more substantially this portion enlarges. This will, to a certain degree, diminish the advantages of the modified c-correction technique. However, considering the 16-day repeat 
cycle of Landsat TM a cloud-free image couple can be found within a time window of approximately three months for most arid, semi-arid and temperate regions of the world ( $\mathrm{Ju}$ and Roy, 2008). If this condition is fulfilled, even when illumination differences are slightly more extreme than in our case, we can suppose that the advantages of the modified ccorrection technique will still outweigh its disadvantages. This prerequisite is often not feasible for tropical and high-latitude regions (Marshall et al., 1994; Sano et al., 2007; Ju et al., 2008). Latitude is an important factor because of two reasons. Firstly, the latitude of the study area determines its macro-climate and as a consequence the frequency of cloud-free remote sensing conditions. Secondly, the latitude of the area of interest also determines the magnitude of the potential maximal difference in illumination. This magnitude increases with increasing latitude. For tropical regions, differences in illumination throughout the year are relatively small. Therefore, in favor of finding a cloud-free image couple, the time window can possibly be extended, assuming that the phenology issue does not distort the analysis. For high-latitude zones both cloudiness and illumination conditions hamper the set up of a bitemporal change detection scheme (Marshall et al., 1994; Ju and Roy, 2008; Verbyla et al., 2008), although the disadvantage of bad weather is in some degree compensated for by acquiring more images because of the bigger sidelap of adjacent frames (Kontoes and Stakenburg, 1990). For these case studies at high latitudes and/or in highly rugged terrain and/or with a substantial deviation from the anniversary date scheme, we suggest authors to make a rapid comparison between the original and modified c-correction technique to assess which approach most adequately removes illumination effects. This task can be easily accomplished without the need of any additional data by applying the optimality concept as described in this paper.

In summary, correcting for illumination effects is especially important in change detection studies conducted in rugged terrain with highly differing illumination conditions imagery. Fire severity applications are affected more than other remote sensing studies since it is important to acquire the first available cloud-free post-fire image and as a consequence a potential divergence from the ideal anniversary date acquisition scheme may exist. The modified c-correction technique resulted in a more reliable spectral indices based change detection study in rugged terrain with two images that deviated up to two months from the ideal anniversary date scheme.

\section{Conclusions}

Based on the spectral index theory, the effect of illumination on the dNBR optimality for assessing fire severity using pre- (2006) and post-fire (2007) Landsat TM imagery was evaluated for the 2007 Peloponnese wildfires. South and south-east exposed slopes obtained higher optimality values than north and north-west facing slopes. The better a pixel was illuminated in 2006 and 2007, the higher the dNBR optimality was. Apart from the average illumination condition, also the difference in illumination geometry between the images contributed to variations in index optimality: a better optimality was achieved with smaller differences in illumination.

To improve the performance of the index, the c-correction method and a modified version of this technique were applied to derive terrain corrected reflectance. Opposed to the original ccorrection method that normalizes to an intermediate illumination condition, the modified ccorrection technique corrects towards maximum illumination conditions. The original ccorrection method improved the optimality of badly illuminated pixels while deteriorating the optimality of well illuminated pixels. The modified c-correction technique neutralized this feature while retaining the prime characteristic of topographic correction techniques, i.e. 
detrending the illumination-reflectance relationship. The resulting median dNBR optimality scores of topographically uncorrected, c-corrected and modified c-correction data were respectively $0.58,0.60$ and 0.71 . It should be noted that for a minority of pixels that experienced low average illumination and/or high difference in illumination conditions the original c-correction technique outperformed the modified c-correction technique. For case studies in which these circumstances are prevalent, we suggest authors to undertake a rapid evaluation of the original and modified c-correction technique to assess which technique most adequately minimizes illumination effects. This comparison can easily by achieved using the optimality concept. For this case study conducted in a rough topographical area at the Peloponnese (Greece) with a bi-temporal acquisition scheme substantially deviating from the ideal anniversary date scheme the modified c-correction method resulted in a more reliable fire severity assessment.

\section{Acknowledgements}

The study was financed by the Ghent University special research funds (BOF: Bijzonder Onderzoeksfonds). The authors would like to thank the reviewers for their constructive comments on the manuscript.

\section{References}

Arino, O., Brockman, C., Veraini, B. and Pittella, G. s.d. ESA products and processing algorithms for Landsat TM. http://earth.esa.int/ers/sysutil/008e3.html (last visited on 12/07/2009)

Bishop, M. and Colby, J. 2002. Anisotropic reflectance correction of SPOT-3 HRV imagery. International Journal of Remote Sensing, 23, 2125-2131

Bisson, M., Fornaciai, A., Coli, A., Mazzarini, F. and Pareschi, M. 2008. The Vegetation Resilience After Fire (VRAF) index: Development, implementation and an illustration from central Italy, International Journal of Applied Earth Observation and Geoinformation, 10, 312-329

Brewer, K., Winne, C., Redmond, R., Opitz, D. and Mangrich, M. 2005. Classifying and mapping wildfire severity: a comparison of methods. Photogrammetric Engineering \& Remote Sensing, 71, 1311-1320

Chafer, C., Noonan, M. and Macnaught, E. 2004. The post-fire measurement of fire severity and intensity in the Christmas 2001 Sydney wildfires. International Journal of Wildland Fire, $13,227-240$

Chafer, C. 2008. A comparison of fire severity measures: An Australian example and implications for predicting major areas of soil erosion. Catena, 74, 235-245

Chander, G., Markham, L. and Barsi, J. 2007. Revised Landsat-5 Thematic Mapper radiometric calibration. IEEE Geoscience and Remote Sensing Letters, 4, 490-494

Chavez, P. 1996. Image-based atmospheric corrections - revisited and improved. Photogrammetric Engineering \& Remote Sensing, 62, 1025-1036

Chuvieco, E., Englefield, P., Trishchenko, A. and Luo, Y. 2008. Generation of long time series of burn area maps of the boreal forest from NOAA-AVHRR composite data. Remote Sensing of Environment, 112, 2381-2396 
Civco, D. 1989. Topographic normalization of Landsat Thematic Mapper Digital Imagery. Photogrammetric Engineering and Remote Sensing, 55, 1303-1309

Cocke, A., Fule, P. and Crouse, J. 2005. Comparison of burn severity assessments using Differenced Normalized Burn Ratio and ground data. International Journal of Wildland Fire, $14,189-198$

Colby, J. and Keating, P. 1998. Land cover classifications using Landsat TM imagery in the tropical highlands: the influence of anisotropic reflectance. International Journal of Remote Sensing, 19, 1479-1500

Coppin, P., Jonckheere, I., Nackaerts, K. and Muys, B. 2004. Digital change detection techniques in ecosystem monitoring: a review. International Journal of Remote Sensing, 25, $1565-1595$

De Santis, A. and Chuvieco, E. 2009. GeoCBI: a modified version of the Composite Burn Index for the initial assessment of the short-term burn severity from remotely sensed data. Remote Sensing of Environment, 113, 554-562

Diaz-Delgado, R., Lloret, F. and Pons, X. 2003. Influence of fire severity on plant regeneration by means of remote sensing imagery. International Journal of Remote Sensing, $24,1751-1763$

Dwyer, E., Perreira, J., Grégoire, J. and DaCamara, C. 1999. Characterization of the spatiotemporal patterns of global fire activity using satellite imagery for the period April 1992 to March 1993. Journal of Biogeography, 27, 57-69

Eidenshink, J., Schwind, B., Brewer, K., Zhu, Z., Quayle, B. and Howard, S. 2007. A project for monitoring trends in burn severity. Fire Ecology, 3, 3-21

Ekstrand, S. 1996. Landsat TM-based forest damage assessment: correction for topographic effects. Photogrammetric Engineering \& Remote Sensing, 62, 151-161

Epting, J. and Verbyla, D. 2005. Landscape-level interactions of prefire vegetation, burn severity, and postfire vegetation over a 16-year period in interior Alaska, Canadian Journal Forest Research, 35, 1367-1377

Epting, J., Verbyla, D. and Sorbel, B. 2005. Evaluation of remotely sensed indices for assessing burn severity in interior Alaska using Landsat TM and ETM+. Remote Sensing of Environment, 96, 328-339

Escuin, S., Navarro, R. and Fernandez, P. 2008. Fire severity assessment by using NBR (Normalized Burn Ratio) and NDVI (Normalized Difference Vegetation Index) derived from LANDSAT TM/ETM images. International Journal of Remote Sensing, 29, 1053-1073

Fuller, M., Groom, G. and Wallis, S. 1994. The availability of Landsat TM images of Great Britain. International Journal of Remote Sensing, 15, 1357-1362

Garcia, M. and Chuvieco, E. 2004. Assessment of the potential of SAC-C/MMRS imagery for mapping burned areas in Spain. Remote Sensing of Environment, 92, 414-423

Giglio, L., Csiszar, I, Restas, A., Morisette, J., Schroeder, W., Morton, D. and Justice, C. 2008. Active fire detection and characterization with advanced spaceborne thermal emission and reflection radiometer (ASTER), Remote Sensing of Environment, 112, 3055-3063 
Gonzalez-Alonso, F., Merino-De-Miguel, S., Roldan-Zamarron, A., Garcia-Gigorro, S. and Cuevas, J. 2007. MERIS Full Resolution data for mapping level-of-damage caused by forest fires: the Valencia de Alcántara event in August 2003. International Journal of Remote Sensing, 28, 789-809

Hammill, K. and Bradstock, R. 2006. Remote sensing of fire severity in the Blue Mountains: influence of vegetation type and inferring fire intensity. International Journal of Wildland Fire, 15, 213-226

Hollander, M. and Wolfe, D. 1999. Nonparametric statistical methods. John Wiley \& Sons, New York, 1999

Huang, H., Gong, P., Clinton, N. and Hui, F. 2008. Reduction of atmospheric and topographic effect on Landsat TM data for forest classification. International Journal of Remote Sensing, $29,5623-5642$

Hudak, A., Morgan, P., Bobbitt, M., Smith, A., Lewis, S., Lentile, L., Robichad, P., Clark, J. and McKinley, R. 2007. The relationship of multispectral satellite imagery to immediate fire effects. Journal of Fire Ecology, 3, 64-90

Isaev, A., Korovin, G., Bartalev, S., Ershov, D., Janetos, A., Kasischke, E., Shugart, H., French, N, Orlick, B. and Murphy, T. 2002. Using remote sensing to assess Russian forest fire carbon emissions. Climatic Change, 55, 239-245

Jarvis, A., Reuter, H., Nelson, A. and Geuvara, E. 2006. Hole-filled seamless SRTM data V3, International Centre for Tropical Agriculture (CIAT), available from http://srtm.csi.cgiar.org

Ju, J. and Roy, D. 2008. The availability of cloud-free Landsat ETM+ data over the conterminous United States and globally. Remote Sensing of Environment, 112, 1196-1211

Keeley, J. 2009. Fire intensity, fire severity and burn severity: a brief review and suggested usage. International Journal of Wildland Fire, 18, 116-126

Key, C. 2005. Remote sensing sensitivity to fire severity and fire recovery. In J. De la Riva, F. Perez-Cabello and E. Chuvieco (Editors) Proceedings of the $5^{\text {th }}$ international workshop on remote sensing and GIS applications to forest fire management: fire effects assessment (pp. 29-39). Universidad de Zaragoza.

Key, C. and Benson, N. 2005. Landscape assessment: ground measure of severity; the Composite Burn Index, and remote sensing of severity, the Normalized Burn Index. In D. Lutes, R. Keane, J. Caratti, C. Key, N. Benson, S. Sutherland and L. Gangi (Editors). FIREMON: Fire effects monitoring and inventory system (pp. 1-51). USDA Forest Service, Rocky Mountains Research Station, General Technical Report RMRS-GTR-164-CD LA

Kobayashi, S. and Sanga-Ngoie, K. 2008. The integrated radiometric correction of optical remote sensing imageries. International Journal of Remote Sensing. 29, 5957-5985

Kontoes, C and Stakenburg, J. 1990. Availability of cloud-free Landsat images for operational projects. The analysis of cloud-cover figures over the countries of the European community. International Journal of Remote Sensing, 11, 1599-1608

Landmann, T. 2003. Characterizing sub-pixel Landsat ETM+ fire severity on experimental fire in the Kruger National Park, South Africa. South African Journal of Science, 99, 357-359 
Lee, B., Kim, S., Chung, J. and Park, P. 2008. Estimation of fire severity by use of Landsat TM images and its relevance to vegetation and topography in the 2000 Samcheok forest fire. Journal of Forest Research, 13, 197-204

Lentile, L., Smith, F. \& Shepperd, W. 2005. Patch structure, fire-scar formation, and tree regeneration in a large mixed-severity fire in the South Dakota Black Hills, USA. Canadian Journal Forest Research, 35, 2875-2885

Lentile, L., Holden, Z., Smith, A., Falkowski, M., Hudak, A., Morgan, P., Lewis, S., Gessler, P. and Benson, N. 2006. Remote sensing techniques to assess active fire characteristics and post-fire effects. International Journal of Wildland Fire, 15, 319-345

Leprieur, C., Durand, J. and Peyron, J. 1988. Influence of topography on forest reflectance using Landsat Thematic Mapper and digital terrain data. Photogrammetric Engineering and Remote Sensing, 54, 491-496

Lopez-Garcia, M. and Caselles, V. 1991. Mapping burns and natural reforestation using Thematic Mapper data. Geocarto International, 6, 31-37

Marshall, G., Dowdeswell, J. and Rees, W. 1994. The spatial and temporal effect of cloud cover on the acquisition of high-quality Landsat imagery in the European Arctic sector. Remote Sensing of Environment, 50, 149-160

Meyer, P., Itten, K., Kellenberger, T., Sandmeier, S. and Sandmeier, R. 1993. Radiometric corrections of topographically induced effects on Landsat TM data in an alpine environment. ISPRS Journal of Photogrammetry and Remote Sensing, 48, 17-28

Mitri, G. and Gitas, I. 2004. A performance evaluation of a burned area object-based classification model when applied to topographically and non-topographically corrected TM imagery. International Journal of Remote Sensing, 25, 2863-2870

Murphy, K., Reynolds, J. and Koltun, J. 2008. Evaluating the ability of the differenced Normalized Burn Ratio (dNBR) to predict ecologically significant burn severity in Alaskan boreal forests. International Journal of Wildland Fire, 17, 490-499

Pausas, J. 2004. Changes in fire and climate in the eastern Iberian peninsula (Mediterranean Basin). Climatic Change, 63, 337-350

Pereira, J., Sa, A., Sousa, A., Silva, J., Santos, T. and Carreiras, J. 1999. Spectral characterization and discrimination of burnt areas. in Chuvieco, E. (Editor). Remote sensing of large wildfires in the European Mediterranean Basin. Springer-Verlag, Berlin, pp. 123-138

Polunin, O. 1980. Flowers of Greece and the Balkans. A field guide. Oxford University Press, Oxford, 1980

Riano, D., Chuvieco, E., Salas, J. and Aguado, I. 2003. Assessment of different topographic corrections in Landsat-TM data for mapping vegetation types. IEEE Transaction on Geosciences and Remote Sensing, 41, 1056-1061

Riano, D., Moreno-Ruiz, J., Isidoros, D. and Ustin, S. 2007. Global spatial patterns and temporal trends of burned area between 1981 and 2000 using NOAA-NASA Pathfinder. Global Change Biology, 13, 40-50 
Roy, D., Boschetti, L. and Trigg, S. 2006. Remote sensing of fire severity: assessing the performance of the Normalized Burn Ratio. IEEE Transactions on Geoscience and Remote Sensing, 3, 112-116

Ruiz-Gallardo, J., Castano, S. and Calera, A. 2004. Application of remote sensing and GIS to locate priority intervention areas after wildland fires in Mediterranean systems: a case study from south-eastern Spain. International Journal of Wildland Fire, 13, 241-252

Sano, E., Ferreira, L., Asner, G. and Steinke, E. 2007. Spatial and temporal probabilities of obtaining cloud-free Landsat images over the Brazilian tropical savanna. International Journal of Remote Sensing, 28, 2739-2752

Soenen, S., Peddle, D., Coburn, C., Hall, R. and Hall, F. 2008. Improved topographic correction of forest image data using a 3-D canopy reflectance model in multiple forward mode. International Journal of Remote Sensing, 29, 1007-1027

Song, C. and Woodcock, C. 2003. Monitoring forest succession with multitemporal Landsat images: factors of uncertainty. IEEE Transactions on Geoscience and Remote Sensing, 41, 2557-2567

Stow, D., Petersen, A., Rogan, J. \& Franklin, J. 2007. Mapping burn severity of Mediterranean-type vegetation using satellite multispectral data. GIScience \& Remote Sensing, 44, 1-23

Telesca, L. and Lasaponara, R. 2006. Pre- and post-fire behavioral trends revealed in satellite NDVI time series. Geophysical Research Letters, 31, L14401

Teillet, P., Guindon, B. and Goodenough, D. 1982. On the slope-aspect correction of multispectral scanner data. Canadian Journal of Remote Sensing, 8, 84-106

Verbyla, D., Kasischke, E. and Hoy, E. 2008. Seasonal and topographic effects on estimating fire severity from Landsat TM/ETM+ data. International Journal of Wildland Fire, 17, 527534

Verstraete, M. and Pinty, B. 1996. Designing optimal spectral indexes for remote sensing applications. IEEE Transactions on Geoscience and Remote Sensing, 34, 1254-1265

Vicente-Serrano, S., Perez-Cabello, F. and Lasanta, T. 2008. Assessment of radiometric correction techniques in analyzing vegetation variability and change using time series of Landsat images. Remote Sensing of Environment, 112, 3916-3934

Vincini, M. and Frazzi, E. 2003. Multitemporal evaluation of topographic normalization methods on deciduous forest TM data. IEEE Transactions on Geoscience and Remote Sensing, 41, 2586-2590

Wu, J., Bauer, M., Wang, D. and Manson, S. 2008. A comparison of illumination geometrybased methods for topographic correction of Quickbird images of an undulant area. ISPRS Journal of Photogrammetry \& Remote Sensing, 63, 223-236 
Figure 1. Location of the study area (Landsat TM image 23/07/2007 RGB-743, UTM 34S ED50).

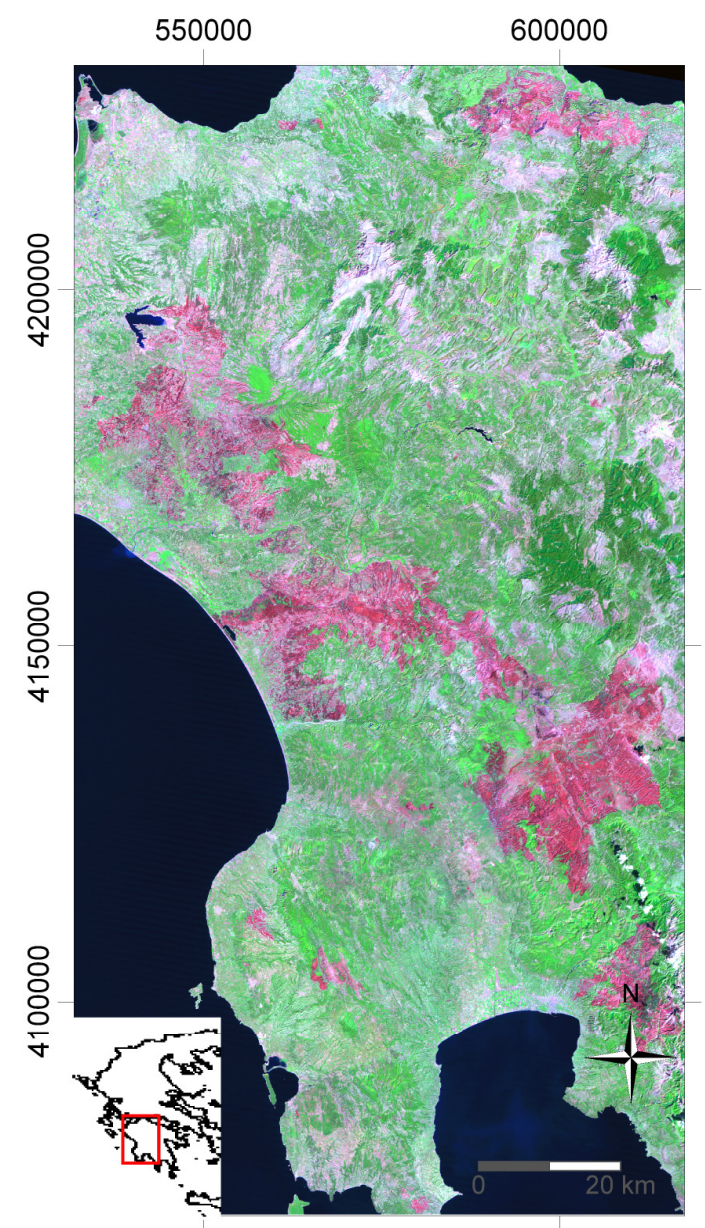




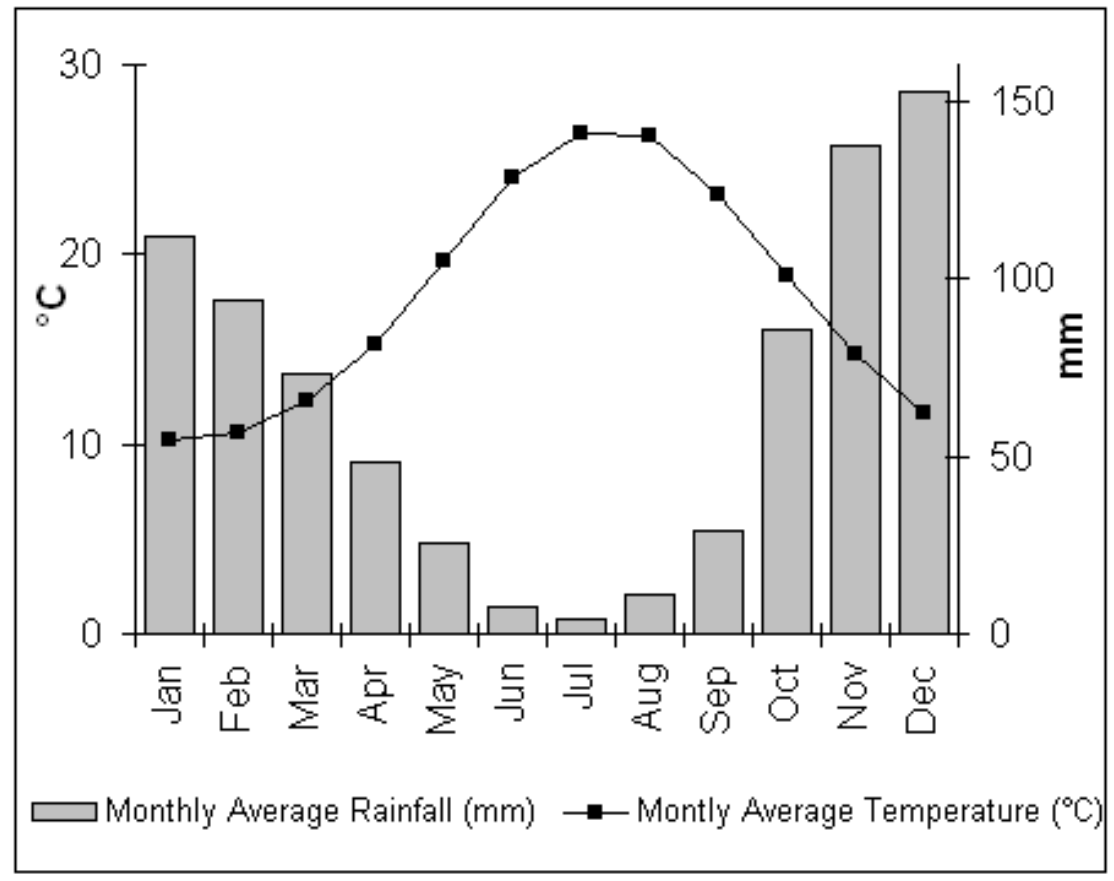

Figure 2. Ombrothermic diagram of the Kalamata (Peloponnese, Greece) meteorological station ( $37^{\circ} 4^{\prime} 1 " \mathrm{~N}$ $22^{\circ} 1$ '1" E) 1956-1997 (Hellenic National Meteorological Service, www.hnms.gr). 


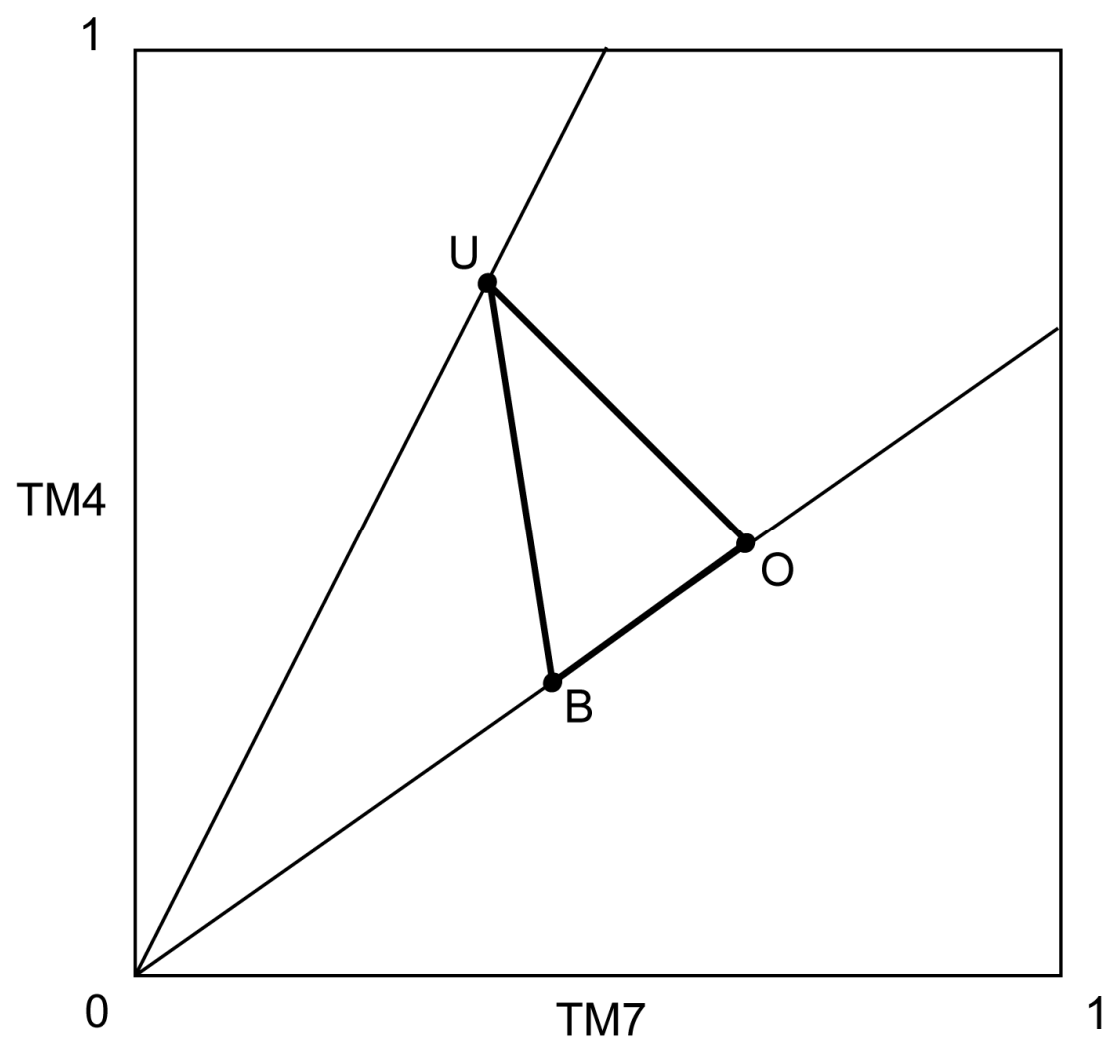

Figure 3. Example pre/post-fire trajectory of a pixel in the TM4-TM7 feature space. A pixel displaces from unburned ( $\mathrm{U})$ to burned (B). O resembles the position of an optimally sensed burned pixel. The dNBR is sensitive to the displacement $|U O|$ and insensitive to the displacement $|O B|$. 
A. uncorrected dNBR optimality map
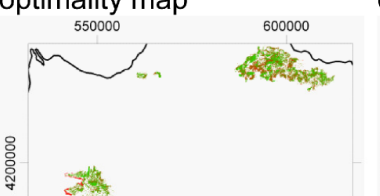

总

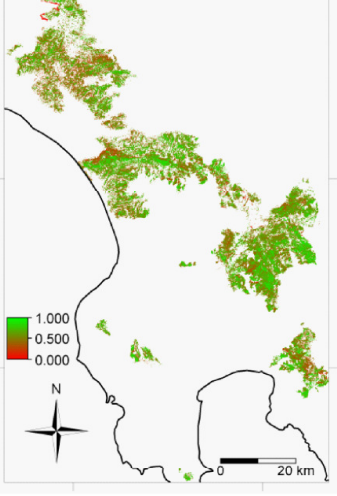

D. uncorrected dNBR optimality histogram

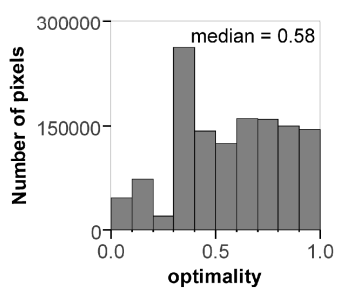

B. c-corrected dNBR optimality map
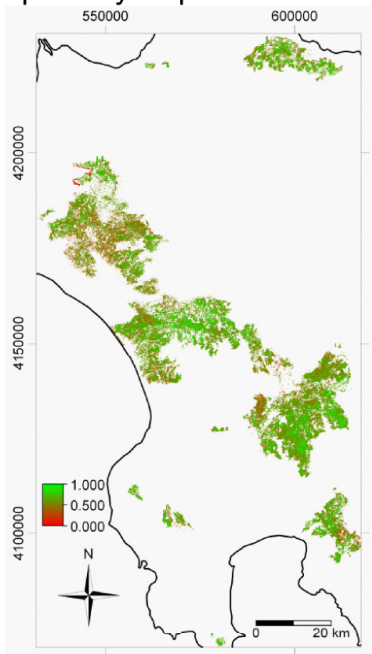

E. c-corrected dNBR optimality histogram

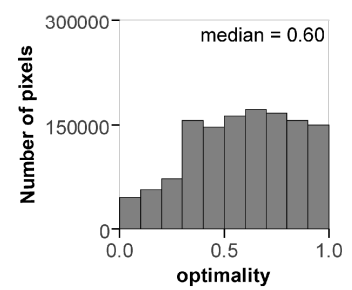

C. modified c-correction dNBR optimality map
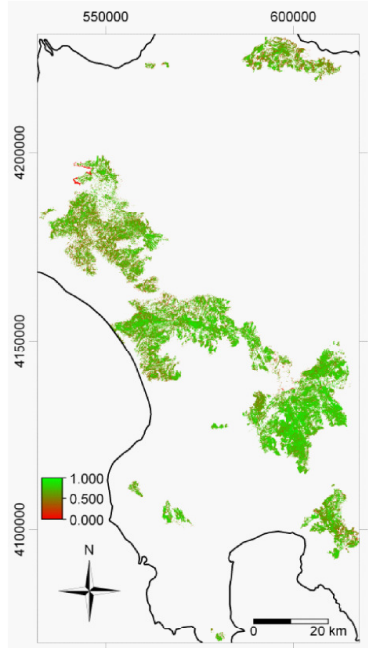

E. modified c-correction dNBR optimality histogram

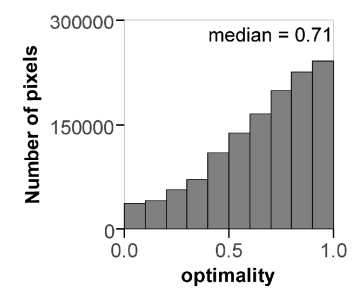

Figure 4. Topographically uncorrected (a and d), c-corrected (b and e) and modified c-correction (c and f) dNBR optimality maps and histograms. 
A. Median uncorrected and corrected dNBR optimality by aspect class

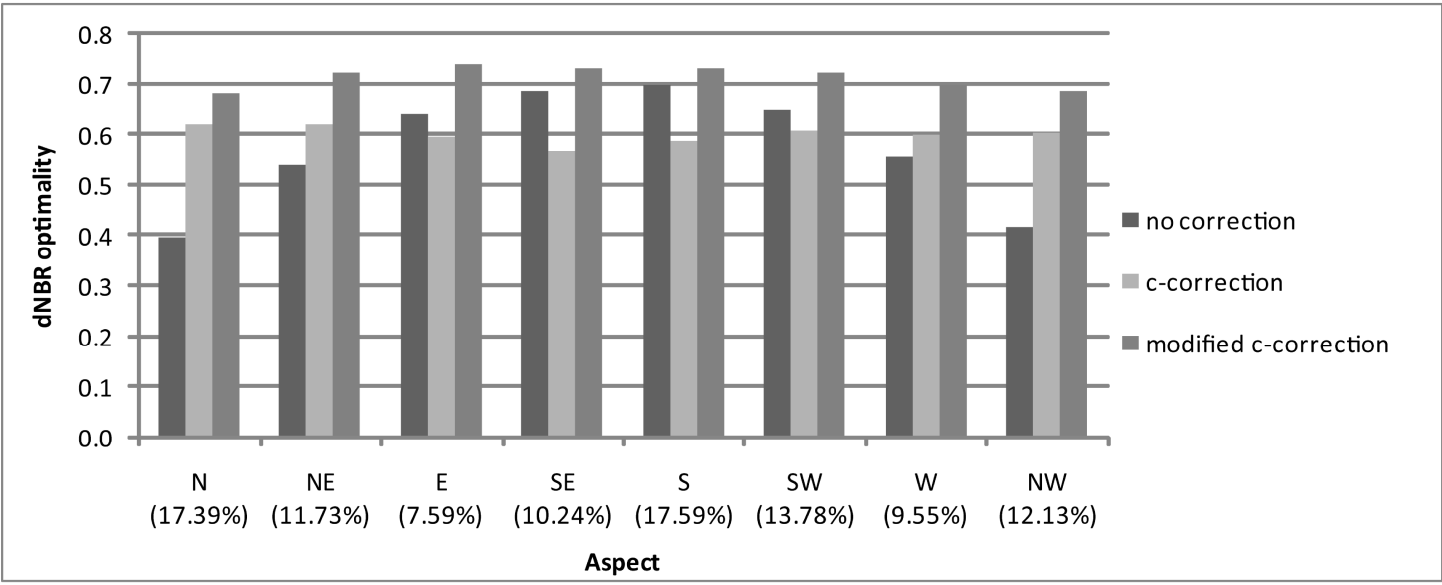

B. Median uncorrected and corrected dNBR optimality by slope class

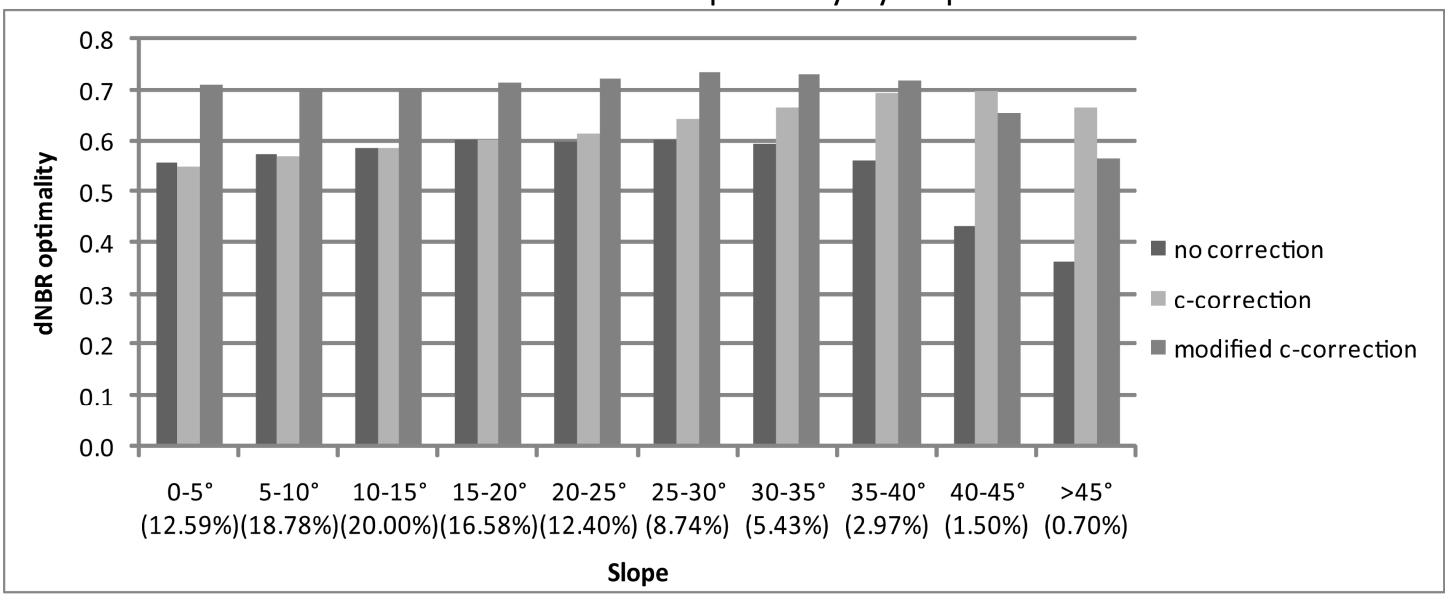

Figure 5. Median topographically uncorrected, c-corrected and modified c-correction dNBR optimality score by aspect (a) and slope (b) class (the percentage between brackets represents the class's percentage of the total number of burned pixels). 
A. Median uncorrected and corrected dNBR optimality by average illumination class

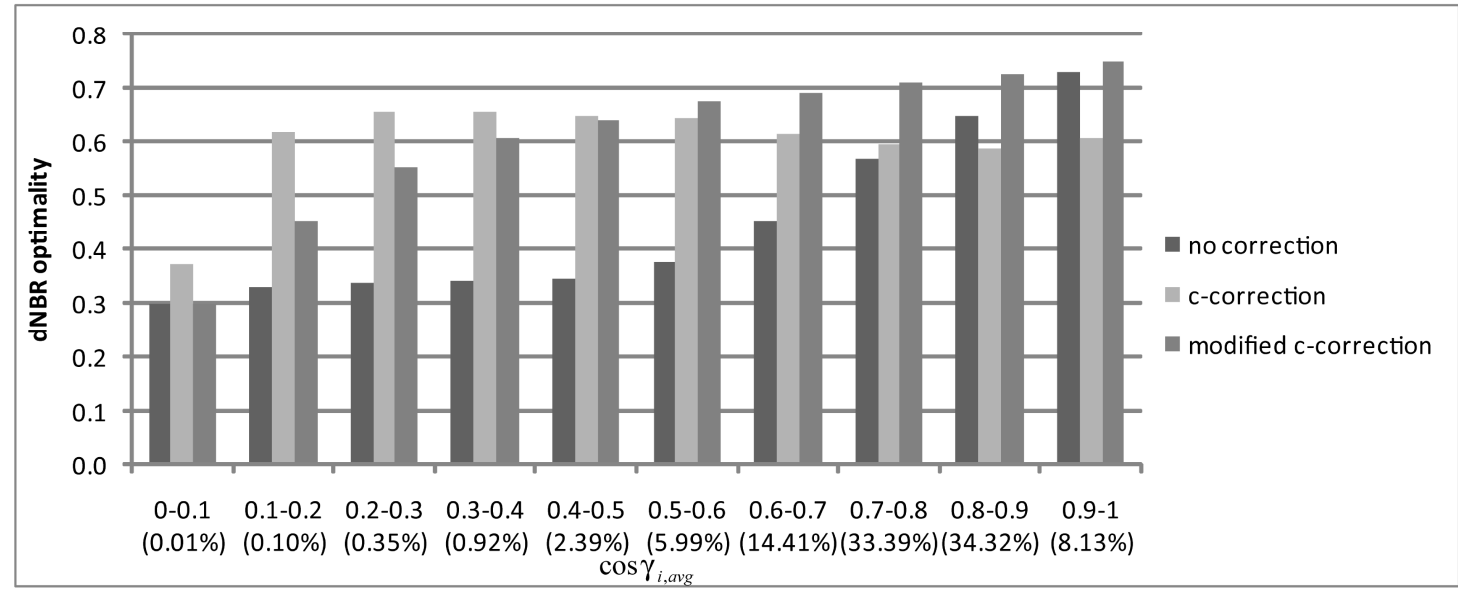

B. Median uncorrected and corrected dNBR optimality by difference in illumination class

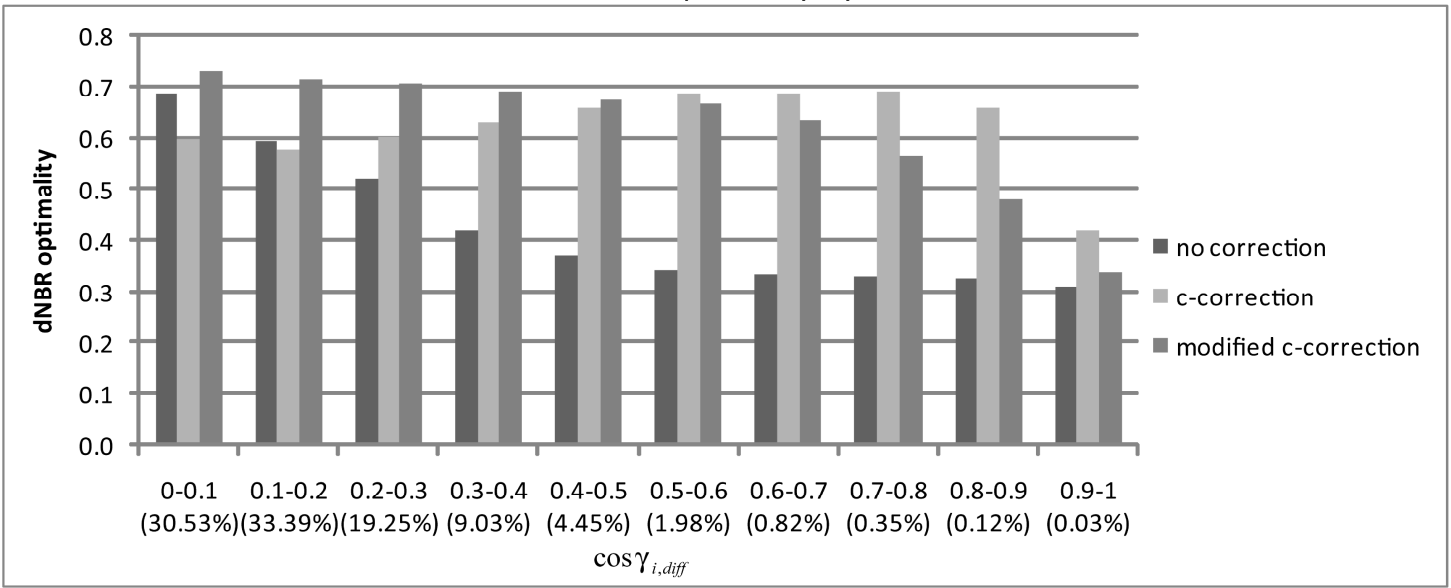

Figure 6. Median topographically uncorrected, c-corrected and modified c-correction dNBR optimality score by average illumination $\cos \gamma_{i, a v g}$ (a) and difference in illumination $\cos \gamma_{i, \text { diff }}$ (b) class (the percentage between brackets represents the class's percentage of the total number of burned pixels). 
Table 1: Regression model results for the model form $\rho=a \cos \gamma_{i}+b$.

Table 1: regression model results for the model form $\rho=a \cos \gamma_{i}+b$

\begin{tabular}{llll}
\hline Image band and correction & $\mathrm{a}$ & $\mathrm{b}$ & $\mathrm{R}^{2}$ \\
\hline 2006 TM4 no topographic correction & 0.0575 & 0.1713 & 0.02 \\
2006 TM4 c-correction & 0.0002 & 0.2224 & $<0.001$ \\
2006 TM4 modified c-correction & 0.0002 & 0.2287 & $<0.001$ \\
2006 TM7 no topographic correction & 0.0436 & 0.0532 & 0.02 \\
2006 TM7 c-correction & -0.005 & 0.0925 & $<0.001$ \\
2006 TM7 modified c-correction & -0.005 & 0.0973 & $<0.001$ \\
2007 TM4 no topographic correction & 0.0912 & 0.0468 & 0.14 \\
2007 TM4 c-correction & 0.0010 & 0.1118 & $<0.001$ \\
2007 TM4 modified c-correction & 0.0012 & 0.1370 & $<0.001$ \\
2007 TM7 no topographic correction & 0.1417 & 0.0423 & 0.29 \\
2007 TM7 c-correction & -0.0010 & 0.1514 & $<0.001$ \\
2007 TM7 modified c-correction & -0.0127 & 0.1930 & $<0.001$ \\
\hline
\end{tabular}

